\title{
Effect of food on the Oral Bioavailability of Berberine and Monacolin Administered in Combination in Healthy Male Volunteers
}

\author{
Stefano Persiani ${ }^{1}$, Federica Sala ${ }^{1}$, Monique Zangarini ${ }^{1}$, Carla Manzotti ${ }^{1}$, Milena Colovic ${ }^{1}$, Yves Donazzolo ${ }^{2}$, \\ Beatrice Barbetta ${ }^{1}$, Cristina Vitalini ${ }^{1}$, Giampaolo Giacovelli ${ }^{1}$, Claudio Benvenuti ${ }^{3}$ and Lucio C. Rovati ${ }^{1}$ \\ 1. R\&D Division, Rottapharm|Biotech S.r.l., Monza 20900, Italy \\ 2. Eurofins/Optimed, Gieres 38610, France \\ 3. Medical Department, Rottapharm|Madaus, Monza 20900, Italy
}

\begin{abstract}
Objectives: To investigate the effect of food on the bioavailability of a combination of monacolin and berberine in healthy volunteers. Methods: Eighteen male volunteers received a single recommended oral dose of the combination under fasted conditions (reference) and fed conditions (high fat meal; test), in a randomized, open label, crossover fashion. Plasma concentrations of berberine, monacolin and its metabolite were measured by LC-MS/MS. Pharmacokinetic parameters were determined by non-compartmental analysis. No effect of food was assumed if the $90 \%$ CIs (confidence intervals) for estimated ratio test/reference was included in the acceptance limits 0.80-1.25 for phenotyping metrics $\mathrm{AUC}_{\mathrm{t}}$ and $C_{\max }$. Key findings: For berberine, the $C_{\max }$ and $\mathrm{AUC}_{\mathrm{t}}$ test/reference ratios were 2.97 and 2.69, respectively, and relevant 90\% CIs (2.25-3.91 and 2.15-3.36, respectively) were above the acceptance limit. For lovastatin hydroxy acid, the active metabolite of monacolin, the test/reference ratios were $1.18\left(C_{\max }\right)$ and $0.98\left(\mathrm{AUC}_{\mathrm{t}}\right.$. The $90 \%$ CIs fell entirely within the acceptance limit for $\mathrm{AUC}_{\mathrm{t}},(0.85-1.13)$, whereas the upper bound of the $90 \%$ CIs for $C_{\max }(1.01-1.37)$ was just above the predefined interval. Conclusions: Food intake significantly increases berberine bioavailability and does not significantly affect monacolin bioavailability when these two extracts are administered in combination.
\end{abstract}

Key words: Berberine, monacolin, food effects, bioavailability, ArmoLIPID PLUS, healthy volunteers.

\section{Introduction}

Hypercholesterolemia is a well-known risk factor for coronary artery, cerebrovascular and peripheral artery diseases $[1,2]$. There is general agreement on the need for therapeutic interventions in the secondary prevention of cardiovascular disease, whereas the cost effectiveness and societal impact of primary prevention therapy remains controversial, being the cost/benefit ratio less favorable [3]. The revised guidelines on dyslipidemia provide expanded recommendations that health behavior interventions remain the cornerstone for the prevention of

Corresponding author: Stefano Persiani, Ph.D., research fields: translational sciences and pharmacokinetics. E-mail: stefano.persiani@rottapharmbiotech.com. cardiovascular disease [4]. However, to be effective, therapeutic life-style changes, including exercise and diet, must be followed without derogations for the whole life, an extremely difficult task in the present society. To achieve this goal, food supplements, containing herbal products that could integrate imperfect therapeutic life-style changes obtaining a satisfactory control of cardiovascular risk factors [5], are becoming increasingly popular.

Use of these products to maintain healthy status has risen considerably and it is estimated that not less than $80 \%$ of the world's population relies on herbal remedies as a moderate approach to healing and as a source of healthcare [6]. Despite this importance and the widespread use, data on the bioavailability of 
herbal extracts are scarse [7], preventing correlation between effects and exposure and thus the optimal consumption of these products.

Berberine hydrochloride and fermented red yeast rice extract (Monascus purpureus) which contains monacolin are the herbal products objective of the present investigation. They are marketed in several European countries not only as single ingredients, but also in combination and they are well described for their lipid-lowering properties [8-10]. Berberine, a natural plant extract from Berberis aristata bark, reduces serum cholesterol by increasing LDL receptor on the liver cell surface, and inhibits triglycerides biosynthesis via the activation of AMP-activated proteokinase [11]. Monacolin, also known as lovastatin lactone, lowers cholesterol by inhibiting the activity of

HMG-CoA (3-hydroxy-3-methylglutaryl-coenzyme A) reductase, the rate limiting step for cholesterol synthesis in the liver [12]. In clinical studies red yeast rice reduced significantly total and LDL cholesterol [13], and berberine improved also triglycerides profile [14]. Further and recent data showed that also their combination significantly improved the lipid profile in subjects with altered cholesterol and/or triglycerides [10], and in subjects intolerant to statins $[15,16]$. In another clinical study [17], the combination showed that the favorable metabolic response could also yield improvements in the cardiovascular risk profile. The study was the first documenting that the combination of berberine and monacolin with dietary counseling decreases the prevalence of metabolic syndrome and reduces the risk of coronary heart disease, calculated using the Framingham Risk Score [17]. The benefits of recommending the combination of berberine and monacolin with diet and lifestyle changes versus diet alone have been further confirmed in another extended multicenter-randomized clinical trial enrolling dyslipidemic subjects [18]. Moreover, a recent clinical study excluded the potential for the combination of berberine and monacolin to generate clinically relevant inhibition of the activities of CYP1A2, 2D6, 2C19, 3A4 and 2C9 [19].

As data on bioavailability and plasma concentrations of berberine and monacolin when they are administered in combination provide important information to establish correlations between these and the clinical effects observed in the aforementioned studies, they were determined in this study.

In addition, since the bioavailability of products administered orally may be affected by the intake of food, current guidelines [20,21] recommend to assess the effects of food on the rate and extent of absorption for oral products. Food effects indeed can have clinically significant consequences, altering bioavailability by several means, including delay in gastric emptying, stimulation of bile flow, changes in gastrointestinal $\mathrm{pH}$, physical or chemical interaction (e.g., non-specific binding, stability, sequestration) [20]. This approach is well known and applied during the different phases of drug development, whereas it is yet poorly considered in the field of herbal products where food effect is very often unknown. Therefore, with the aim to make progress in this area, the objective of the present clinical investigation was to compare the bioavailability of berberine and monacolin administered in combination at recommended dose in fasting condition and after a high fat meal in healthy male volunteers.

\section{Materials and Methods}

\subsection{Materials}

In order to study a marketed combination of berberine and monacolin that is fully characterized for its content (active ingredients and excipients), and for which safety and efficacy data are available from clinical trials aimed at evaluating its lipid-lowering activity, the food supplement ArmoLIPID PLUS, obtained from Rottapharm|Madaus (Monza, Italy), was used in the present investigation. The analytical standards berberine and lovastatin lactone were purchased from Sigma-Aldrich (St. Louis, MO, USA), 
and lovastatin hydroxy acid from Cayman Chemical (Ann Arbor, MI, USA). Solvents were obtained from standard sources and were of HPLC grade.

\subsection{Clinical Study Design}

A single center, randomized, open-label study was conducted in healthy male volunteers in both fasted and fed conditions according to a single-dose, two-treatment, two-period, and two-sequence, crossover design.

The study was carried out at Eurofins Optimed (Gieres, France) in accordance with the Declaration of Helsinki as modified in Seoul (2008), the recommendations on Good Clinical Practice (ICH E6) and any applicable local regulatory requirements (Code de la Santé Publique). The study obtained the approval of the local Ethics Committee (CPP $n^{\circ}$ : 2014-015 B, 26 March 2014) and the national Health Authorities "ANSM", Agence Nationale de Sécurité du Medicament et des produits de santé (140230B-81, 31 March 2014). Prior to initiation of study procedures, all volunteers gave their written informed consent.

\subsection{Study Population}

Eighteen healthy male volunteers, aged between 18 and 45 years and with mean body mass index between 18 and $28 \mathrm{~kg} / \mathrm{m}^{2}$, were included in the study. All subjects were in good health conditions on the basis of medical history, physical examination and blood tests. They were not receiving any known enzyme inducing or inhibiting drugs or herbal products. All volunteers completed the study according to the protocol and were included in the analysis.

\subsection{Dosage, Administration and Plasma Sampling}

Volunteers received orally one tablet (the recommended dose) of the combination containing $500 \mathrm{mg}$ of berberine, $200 \mathrm{mg}$ of red yeast rice extract (Monascus purpureus) equivalent to $3 \mathrm{mg}$ of monacolin, $10 \mathrm{mg}$ of policosanol, $0.2 \mathrm{mg}$ folic acid,
$2.0 \mathrm{mg}$ coenzyme Q10 and $0.5 \mathrm{mg}$ astaxanthin (ArmoLIPID PLUS, Rottapharm|Madaus, Monza, Italy). All treatments were administered in sitting position with $240 \mathrm{~mL}$ of tap water.

Subjects received the combination on day 1 in two different study periods, according to a randomization list, with at least 7 days washout between each period. Under fasting condition (reference period) the administration was after $10 \mathrm{~h}$ fasting, whereas in fed conditions (test period) the administration occurred within 5 minutes after the completion of the standardized high fat meal that was consumed in no more than 25 minutes. The subjects refrain from food for at least 4 hours after dosing. The composition of the standardized high-fat meal contained approximately $870 \mathrm{kcal}$ with $16 \%$ from proteins, $58 \%$ from fat and $26 \%$ from carbohydrates, as recommended by current guidelines [20, 21].

The sampling times for determinations in plasma of berberine, monacolin and its metabolite (lovastatin hydroxy acid) were: pre dose, 0.5, 1, 1.5, 2, 3, 4, 6, 8, 12,16 and $24 \mathrm{~h}$. Samples were kept at $-80^{\circ} \mathrm{C}$ pending analysis.

\subsection{Bioanalytical Methods}

Two different bioanalytical methods were developed and qualified at Eurofins ADME Bioanalyses (Vergeze, France) to determine the plasma concentrations of berberine, monacolin and its metabolite.

Berberine plasma samples were spiked with ${ }^{13} \mathrm{C}$, ${ }^{2} \mathrm{H}_{3}$-berberine as IS (internal standard) and a plasma protein precipitation was performed using methanol. The supernatant was evaporated, redissolved and injected into the HPLC system (Shimadzu Corporation, Kyoto, Japan) interfaced to a triple quadrupole mass spectrometer (API5500 Qtrap System, AB Sciex Framingham, MA). Chromatographic separation was obtained using a Zorbax SB-C8 column, $5 \mu \mathrm{m}, 2.1 \times 50 \mathrm{~mm}$ (Agilent Technologies, Santa Clara, CA) with the mobile phase 
A consisting of $10 \mathrm{mM}$ ammonium formiate $\mathrm{pH} 3.5$, and the mobile phase $B$ consisting of acetonitrile/methanol (60/40). Data were acquired in the SRM (selected reaction monitoring) mode and the mass spectrometer was set to monitor berberine in the positive ion mode. The monitored transition was $336.1>320.2 \mathrm{~m} / \mathrm{z}$.

Monacolin (lovastatin lactone) and its metabolite (lovastatin hydroxy acid) plasma samples were spiked with the corresponding stable labelled compounds (IS), and acetonitrile was added to obtain a plasma protein precipitation. After centrifugation, the compounds were separated on a Zorbax SB-C18 column, $5 \mu \mathrm{m}$, $2.1 \times 50 \mathrm{~mm}$ (Agilent Technologies, Santa Clara, CA) and injected into the HPLC-MS/MS system (Shimadzu and API5500 Qtrap System). The mobile phases were $10 \mathrm{mM} \mathrm{pH} 5.5$ ammonium acetate, and acetonitrile. The MS/MS system was set to monitor monacolin in the positive ion mode and lovastatin hydroxy acid in the negative ion mode. The monitored transitions were $422.2>199.2$ and $421.2>101.0 \mathrm{~m} / \mathrm{z}$ for monacolin and lovastatin hydroxy acid, respectively.

The two developed methods were qualified. The qualification comprised the determination of linearity on three different runs, the inter- and intra-run precision and accuracy for four concentration levels, the evaluation of methods' specificity and the assessment of storage stability.

\subsection{Pharmacokinetic Analysis}

Pharmacokinetic parameters of berberine, monacolin and lovastatin hydroxy acid were determined from measured plasma concentrations using Kinetica version 4.3 (Thermo Electron Corporation, Philadelphia, USA) and applying a non-compartmental analysis. The maximum plasma concentration $\left(C_{\max }\right)$ and the time to reach it $\left(t_{\max }\right)$ were obtained directly from the plasma concentration-time data. The area under the plasma concentration-time curve from time 0 to the last measurable time point $\left(\mathrm{AUC}_{\mathrm{t}}\right)$ was calculated according to the linear trapezoidal rule. The AUC from time 0 extrapolated to infinite time (AUCinf) was calculated by the equation:

$$
\mathrm{AUCinf}=\mathrm{AUCt}+\mathrm{Ct} / \mathrm{Ke}
$$

Where, $\mathrm{Ct}$ is the last quantifiable concentration and $\mathrm{Ke}$ is the slope of the regression line of the terminal phase of the plasma concentration-time curve. The elimination half-life (t1/2) was calculated by the equation:

$$
\mathrm{t} 1 / 2=0.693 / \mathrm{Ke} .
$$

\subsection{Sample Size Determination and Statistical Analysis}

Intraindividual $\mathrm{CV}_{\mathrm{s}} \%$ were assumed not to exceed $20 \%$ for $C_{\max }$ and $\mathrm{AUC}_{\mathrm{t}}$ for both herbal extracts, allowing the assessment of the food effect with a sample size of 16 subjects [22]. To account for dropouts, 18 subjects were enrolled.

All statistical analyses were performed using SAS versions 9.3 (SAS Inc., Cary, NC, USA).

Log-transformed phenotyping metrics $\mathrm{C}_{\max }$ and $\mathrm{AUC}_{\text {last }}$ were analyzed using PROC MIXED (SAS statistical package, SAS institute - Version 9.3) with treatment, sequence, period and subject-within sequence as fixed terms. The $90 \%$ CIs (confidence intervals) of logarithmic difference between test (fed condition) and reference treatment (fasted condition) were constructed based on the MSE (mean square error) from the ANOVA model. The CIs were then back transformed to obtain the CIs for the ratio fed/fasted of the original scale. According to regulatory guidelines [20, 23], absence of food effect on bioavailability is established if the $90 \%$ CIs for the ratio of the geometric means between fed and fasted treatments, based on log-transformed data, is contained in the equivalence limits of 0.8 to 1.25 for $\mathrm{AUC}_{\mathrm{t}}$ and $C_{\max }$. Differences in $\mathrm{t}_{\max }$ between test and reference treatment were evaluated according to a non-parametric approach, by means of the Wilcoxon signed-rank test for paired samples. Statistical significance was set at $p \leqslant 0.05$. 


\section{Results}

\subsection{Study Population}

Eighteen healthy male subjects completed the study according to the protocol and were included in the analysis. Their mean values of age and body mass index were $27.9 \pm 5.8$ years and $22.93 \pm 2.90 \mathrm{~kg} / \mathrm{m}^{2}$, respectively. Demographic characteristics of the volunteers categorized into group Fed-Fasted and Fasted-Fed are summarized in Table 1. The dose regimen was well tolerated in all volunteers. No TEAEs (treatment emergent adverse events) were reported by the enrolled volunteers during the study.

\subsection{Bioanalytical Methods}

The ranges, precision and accuracy values for the calibration standards are presented in Table 2. Results obtained from the analysis of quality controls ranges were within the acceptable limits, validating the concentrations of berberine, monacolin and its metabolite determined in plasma. The specificity of the methods was tested by the analysis of six different individual batches of blank human plasma spiked with analytes at LLOQ (lower limit of quantification), and then processed and compared to the corresponding blank plasma samples. No interference peaks were detected.

All samples were analysed within the long-term stability period.

\subsection{Pharmacokinetic Analysis}

Mean concentration-time profiles of berberine and lovastatin hydroxy acid, the active metabolite of monacolin, after the administration of the combination of berberine and monacolin in fed and fasted conditions are shown in Fig. 1. The corresponding PK parameters are shown in Table 3.

Berberine plasma concentrations were still measurable $24 \mathrm{~h}$ post-dosing, both in fasting and fed conditions. However, the presence of multiple peaks in the plasma concentration-time profile, indicating an enterohepatic circulation for berberine, as observed in other previous investigations [24, 25], prevented the calculation of $K_{e}$ (elimination rate constant), and thereby of $\mathrm{AUC}_{\mathrm{inf}}$ and $t_{1 / 2}$, (half-life). Significant differences between fed and fasted conditions were observed in the plasma concentration-time profile of berberine. The mean $C_{\max }$ and $\mathrm{AUC}_{\mathrm{t}}$ values were

Table 1 Summary of demographic data for the subjects participating in the study.

\begin{tabular}{lllll}
\hline \multirow{2}{*}{ Demography } & Statistics & Fed-fasted & Fasted-fed & Overall \\
\cline { 3 - 5 } & & $(\boldsymbol{N}=\mathbf{9})$ & $(\boldsymbol{N}=\mathbf{9})$ & $(\boldsymbol{N}=\mathbf{1 8})$ \\
\hline Male & $N(\%)$ & $9(100.0)$ & $9(100.0)$ & $18(100.0)$ \\
Caucasian & $N(\%)$ & $9(100.0)$ & $9(100.0)$ & $27.9 \pm 5.8$ \\
Age $($ years $)$ & Mean \pm SD & $28.3 \pm 7.9$ & $27.4 \pm 3.0$ & $18 / 27.5 / 41$ \\
& Min/median/max & $18 / 29.0 / 41$ & $24 / 27.0 / 34$ & $179.44 \pm 4.09$ \\
Height $(\mathrm{cm})$ & Mean $\pm \mathrm{SD}$ & $180.56 \pm 2.70$ & $178.33 \pm 5.05$ & $171 / 180 / 188$ \\
& Min/median/max & $177 / 181 / 185$ & $171 / 180 / 188$ & $73.80 \pm 9.20$ \\
Weight $(\mathrm{kg})$ & Mean $\pm \mathrm{SD}$ & $75.54 \pm 9.13$ & $72.06 \pm 9.48$ & $57.7 / 76.1 / 85.4$ \\
& Min/median/max & $57.7 / 76.3 / 84.9$ & $59.0 / 70.6 / 85.4$ & $22.93 \pm 2.90$ \\
BMI $\left(\mathrm{kg} / \mathrm{m}^{2}\right)$ & Mean $\pm \mathrm{SD}$ & $23.16 \pm 2.70$ & $22.71 \pm 3.24$ & $18.0 / 23.6 / 26.6$ \\
\hline
\end{tabular}

Table 2 Ranges, inter-assay precision and accuracy values for each compound calibration standards.

\begin{tabular}{llll}
\hline Drug & $\begin{array}{l}\text { Calibration range } \\
(\mathrm{ng} / \mathrm{mL})\end{array}$ & $\begin{array}{l}\text { Inter-assay precision } \\
(\mathrm{CV} \%)\end{array}$ & $\begin{array}{l}\text { Accuracy } \\
(\%)\end{array}$ \\
\hline Berberine & $0.02-5.00$ & $4.52-10.57$ & -8.08 to 5.88 \\
Monacolin (Lovastatin lactone) & $0.1-50.0$ & $0.71-3.67$ & -1.62 to 3.06 \\
Monacolin metabolite (Lovastatin hydroxy acid) & $0.1-50.0$ & $0.80-3.10$ & -7.00 to 5.60 \\
\hline
\end{tabular}



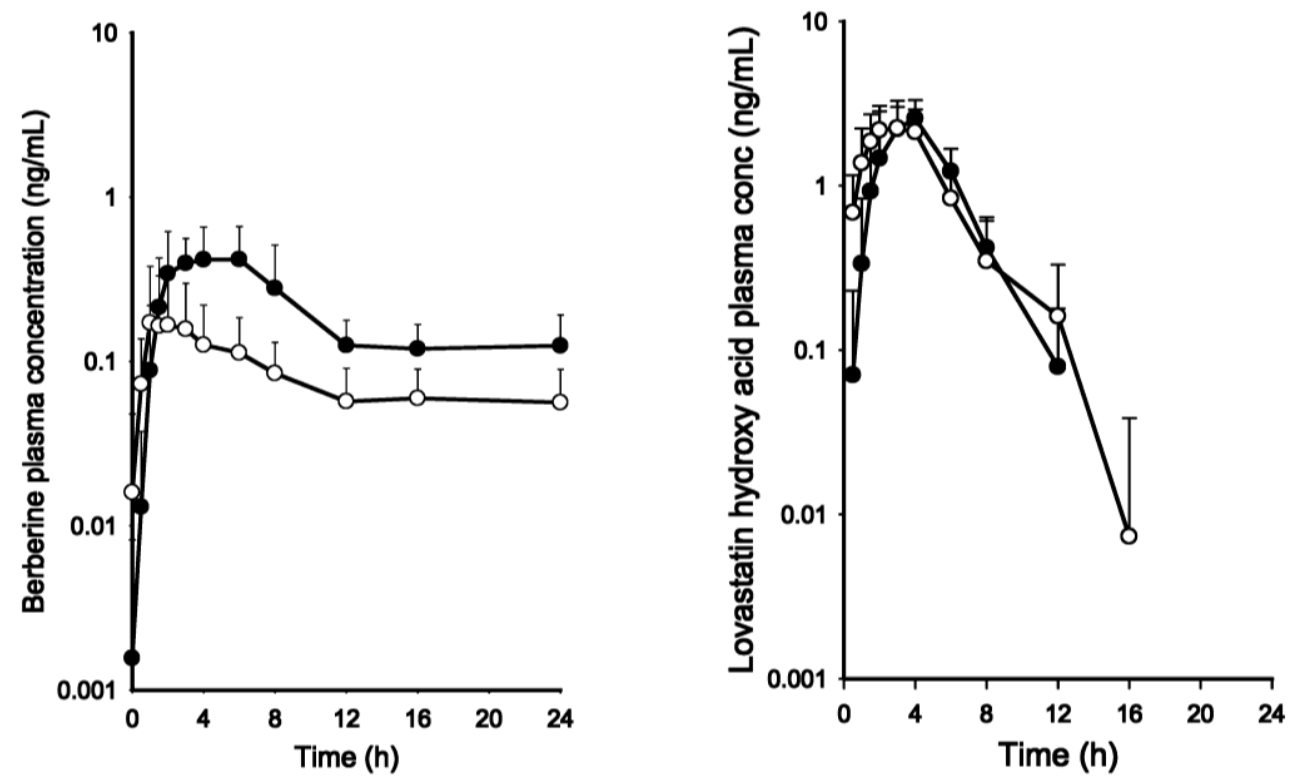

Fig. 1 Mean $( \pm$ SD) plasma concentration-time profile of berberine and lovastatin hydroxy acid in fed condition ( $\bullet)$ and fasted condition $(\circ)$ in healthy subjects.

Table 3 Mean ( \pm SD) pharmacokinetic parameters, fed (test) to fasted condition (reference) GMR (geometric mean ratios), and the $90 \%$ confidence intervals $(90 \% \mathrm{CIs})$.

\begin{tabular}{|c|c|c|c|c|c|c|}
\hline \multicolumn{2}{|c|}{ PK parameters } & Fasted $(N=18)$ & Fed $(N=18)$ & GMR & $90 \% \mathbf{C I}$ & $\mathrm{CV}_{\text {intra }} \%$ \\
\hline & & \multicolumn{5}{|l|}{ Berberine } \\
\hline$C_{\max }$ & $(\mathrm{ng} / \mathrm{mL})$ & $0.24 \pm 0.2$ & $0.59 \pm 0.3$ & 2.97 & $(2.25,3.91)$ & 50.05 \\
\hline $\mathbf{A U C}_{\mathbf{t}}$ & $(\mathrm{ng} / \mathrm{mL} \cdot \mathrm{h})$ & $1.97 \pm 1.2$ & $4.82 \pm 2.1$ & 2.69 & $(2.15,3.36)$ & 39.95 \\
\hline \multirow[t]{2}{*}{$t_{\mathbf{m a x}^{*}}$} & (h) & $2(0.5-6)$ & $4(2-8)$ & NS & & \\
\hline & & \multicolumn{5}{|c|}{ Monacolin (Lovastatin lactone) } \\
\hline$C_{\max }$ & $(\mathrm{ng} / \mathrm{mL})$ & $1.03 \pm 0.5$ & $0.83 \pm 0.4$ & & & \\
\hline \multirow[t]{2}{*}{$t_{\max }$} & (h) & $1(0.5-2)$ & $2.5(1-4)$ & $p<0.05^{* *}$ & & \\
\hline & & \multicolumn{5}{|c|}{ Monacolin Metabolite (Lovastatin hydroxy acid) } \\
\hline$C_{\max }$ & $(\mathrm{ng} / \mathrm{mL})$ & $2.45 \pm 0.8$ & $2.83 \pm 0.8$ & 1.18 & $(1.01,1.37)$ & 26.55 \\
\hline $\mathbf{A U C}_{\mathbf{t}}$ & $(\mathrm{ng} / \mathrm{mL} \cdot \mathrm{h})$ & $12.0 \pm 4.6$ & $11.3 \pm 3.3$ & 0.98 & $(0.85,1.13)$ & 24.22 \\
\hline$t_{\max }$ & (h) & $3(1-4)$ & $4(1.5-6)$ & $p<0.05^{* *}$ & & \\
\hline
\end{tabular}

NS not significant; *tabulated values are median (range); **Wilcoxon signed-rank test.

increased under fed condition from $0.24 \mathrm{ng} / \mathrm{mL}$ to $0.59 \mathrm{ng} / \mathrm{mL}$ and from $1.97 \mathrm{ng} / \mathrm{mL}^{*} \mathrm{~h}$ to $4.82 \mathrm{ng} / \mathrm{mL} \cdot \mathrm{h}$, respectively (approximately $150 \%$ increase). Moreover, the median $t_{\max }$ was delayed by 2 hours under fed condition compared with fasting condition.

For monacolin, only the $C_{\max }$ was determined, since its plasma concentrations decreased rapidly and were often below the limit of quantification. The mean $C_{\max }$ values resulted similar under fasted and fed condition (1.03 ng/mL and $0.83 \mathrm{ng} / \mathrm{mL}$, respectively). Under fasted condition median $t_{\max }$ was $1 \mathrm{~h}$ compared to 2.5 h under fed condition.
Lovastatin hydroxy acid plasma concentrations were above the LLOQ under fasted condition until 16 hours post-dosing, while in fed condition the metabolite was measurable until 12 hours post dosing. The mean peaks plasma concentrations were 2.45 $\mathrm{ng} / \mathrm{mL}$ and $2.83 \mathrm{ng} / \mathrm{mL}$ under fasted and fed condition, respectively. The median $t_{\max }$ was $3 \mathrm{~h}$ under fasted condition and $4 \mathrm{~h}$ under fed condition. $\mathrm{AUC}_{\mathrm{t}}$ mean values were $12.0 \mathrm{ng} / \mathrm{mL} \cdot \mathrm{h}$ and $11.3 \mathrm{ng} / \mathrm{mL} \cdot \mathrm{h}$ under fasted and fed condition, respectively.

The rapid decrease of monacolin and lovastatin hydroxy acid plasma concentrations, due to their short 
half-life of approximately 2 hours [26], prevented the calculation of $K_{e}$, and therefore $\mathrm{AUC}_{\mathrm{inf}}$, and $t_{1 / 2}$, for monacolin and its metabolite.

\subsection{Statistical Analysis}

A summary of the statistical analysis results is reported in Table 3.

For berberine, the $C_{\max }$ and $\mathrm{AUC}_{\mathrm{t}}$ test to reference GMR (geometric mean ratio) were 2.97 and 2.69, respectively, and relevant $90 \%$ CIs were outside (2.25-3.91 and 2.15-3.36, respectively) the equivalence limit of $0.80-1.25$. For monacolin, no statistical analysis was conducted, whereas for lovastatin hydroxy acid, the test to reference GMR was 1.18 and 0.98 for $C_{\max }$ and $\mathrm{AUC}_{\mathrm{t}}$, respectively. The $90 \%$ CIs fell entirely within the equivalence limit for $\mathrm{AUC}_{\mathrm{t}}(0.85-1.13)$, whereas the upper bound of the 90\% CIs for $C_{\max }(1.01-1.37)$ was just above the threshold of 1.25 .

\section{Discussion}

The objective of the present study represents a relevant topic in the field of herbal products where oral bioavailability data are very often unknown [7], even for standardized and pharmacologically investigated herbal extracts [27]. Many herbal products are currently commercially available and their use continues to increase, but, even though the bioavailability is a key step regarding their health claims [28], the published studies are scarse. As a consequence, most of the herbal products on the market are not adequately characterized and their safe and rational use continues to be a major public health concern [6]. To allow the correlation between effects and exposure of herbal products is essential to investigate their bioavailability in rigorously conducted clinical trials [28]. This study was, therefore, conducted to make progress in this field using marketed herbal extracts and assessing the oral bioavailability of berberine and monacolin when they are administered in combination under fasting conditions and after a standardized high fat meal in healthy male volunteers.

The results, obtained by qualified analytical methods, demonstrated that berberine and monacolin are absorbed and are available to the systemic circulation. Berberine and monacolin, reached peak plasma concentrations at 2 and $1 \mathrm{~h}$ post dosing, respectively. A reduction in the rate of absorption was observed when the combination of berberine and monacolin was taken with food, delaying the median $t_{\max }$ by $2-2.5 \mathrm{~h}$. The plasma concentrations decreased thereafter and monacolin was rapidly eliminated, whereas berberine was still measurable at 24 hours post-dosing. As suggested by current guideline [23], due to the very low plasma concentrations of monacolin, the statistical analysis was conducted only for berberine and lovastatin hydroxy acid, the active metabolite of monacolin.

In terms of exposure $\left(\mathrm{AUC}_{\mathrm{t}}\right)$, the $90 \%$ CIs of geometric mean ratio of lovastatin hydroxy acid was completely included within the predefined no-effect range, indicating no statistical differences in lovastatin exposure between fed and fasted states. Only small changes were observed for $C_{\max }$ value of lovastatin hydroxy acid when the combination of berberine and monacolin was administered with food. The peak plasma concentrations under fed conditions were slightly greater $(16 \%)$ than those measured under fasted conditions, and the upper value of $90 \%$ CIs exceeded the predefined limit (1.37 vs 1.25). However, taking into account that in the present study the inter-subjects variability calculated for $C_{\max }$ was $29 \%$ (data not shown), the $16 \%$ increase due to the food can be considered negligible, being lower than the inter-subjects variability. It is also commonly accepted that single concentrations, in particular extreme concentrations like $C_{\max }$, generally have a larger variation than integrated characteristics like AUC [29]. Given this limited observed effect of food on the rate of bioavailability of lovastatin hydroxy acid and the lack of any effect of the extent of bioavailability, it 
can be reasonably concluded that the pharmacokinetics of the active metabolite of monacolin is not affected by the presence of food and that small changes, if any, in the peak exposure are unlikely to be clinically relevant.

In contrast, a significant food effect was observed on the bioavailability of berberine; a high fat meal increased approximately 2.5 folds the rate and the systemic exposure of berberine in comparison to fasted conditions. The ratios test (fed)/reference (fasted) differed from unity both for $C_{\max }$ and $\mathrm{AUC}_{\mathrm{t}}$ and the corresponding 90\% CIs (2.25-3.91 and 2.15-3.36, respectively) exceeded the accepted limits to exclude a food interaction.

Therefore, the data indicated that administration of the combination of berberine and monacolin with a high fat meal results in an increased exposure to berberine, but not to monacolin. Although the clinical relevance of the increased berberine bioavailability with food warrants investigations in further studies, the concomitant intake of the combination of berberine and monacolin with food should not result in reduced efficacy.

As far as safety is concerned, in a previous study [30], 56 patients were enrolled and were orally treated with $1.2 \mathrm{~g} /$ day of berberine for two weeks to investigate the relationship between clinical effects of berberine and its concentrations. The plasma concentrations of berberine measured in this latter study ranged from 70 to $190 \mathrm{ng} / \mathrm{mL}$ and no apparent side effects were observed. These concentrations were hundreds of folds higher than the concentrations measured after the administration of the combination investigated in the present study with food. Therefore, the increased exposure to berberine when it is administered as a combination with monacolin at the recommended dose under fed conditions, is considered unlikely to pose any safety concerns.

Even thought the combination of berberine and monacolin used in this study is well standardized and pharmacologically investigated, a possible limitation of the present study is that the findings may be applicable only to this specific combination. In fact, current guideline [21] discourages the extrapolation of results from one herbal product to another, because herbal remedies are often a combination of extracts or natural products and their bioavailability might be affected by the extraction process, presence of other components and/or excipients that may differ from one producer to another [21,31]. Nevertheless, this study provides data, such as sample-size, intra-subjects variability and wash out period, which may be useful for the design of future and different clinical trials in the field of herbal products.

\section{Conclusions}

The present clinical study demonstrates that berberine and monacolin after oral administration of their combination at the recommended dose are absorbed and are bioavailable to the systemic circulation in healthy adult male volunteers, providing a benchmark of exposure in support of the observed clinical effects on lipid profile. In addition, it has been demonstrated that the intake of food increases the bioavailability of berberine and does not affect the bioavailability of monacolin when they are administered in combination and in fed conditions.

\section{References}

[1] Smith, S. C. Jr., Smith, S. C. Jr., Allen, J., Blair, S. N., Bonow, R. O., Brass, L. M., Fonarow, G. C., Grundy, S. M., Hiratzka, L., Jones, D., Krumholz, H. M., Mosca, L., Pearson, T., Pfeffer, M. A., and Taubert, K A. 2006. "AHA/ACC Guidelines for Secondary Prevention for Patients with Coronary and Other Atherosclerotic Vascular Disease: 2006 Update Endorsed by the National Heart, Lung, and Blood Institute." Journal of the American College of Cardiology 47 (10): 2130-9.

[2] Baigent, C., Keech, A., Kearney, P. M., Blackwell, L., Buck, G., Pollicino, C., Kirby, A., Sourjina, T., Peto, R., Collins, R., and Simes, R.; Cholesterol Treatment Trialists' (CTT) Collaborators 2005. "Cholesterol Treatment Trialists' Collaborators. Efficacy and safety of Cholesterol-Lowering Treatment: Prospective Meta-analysis of Data from 90.056 Participants in 14 Randomised Trials of Statins." Lancet 366 (9493): 
1267-78.

[3] Genest, J., McPherson, R., Frohlich, J, Anderson, T., Campbell, N., Carpentier, A., Couture, P., Dufour, R., Fodor, G., Francis, G. A., Grover, S., Gupta, M., Hegele, R. A., Lau, D. C., Leiter, L., Lewis, G. F., Lonn, E., Mancini, G. B., Ng, D., Pearson, G. J., Sniderman, A., Stone, J. A., and Ur, E. 2009. "Canadian Cardiovascular Society/Canadian Guidelines for the Diagnosis and Treatment of Dyslipidemia and Prevention of Cardiovascular Disease in the Adult-2009 Recommendations." The Canadian Journal of Cardiology 25 (10): 567-79. Review.

[4] Anderson, T. J., Grégoire, J., Hegele, R. A., Couture, P., Mancini, G. B., McPherson, R., Francis, G. A., Poirier, P., Lau, D. C., Grover, S., Genest, J. Jr., Carpentier, A. C., Dufour, R., Gupta, M., Ward, R., Leiter, L. A., Lonn, E., Ng, D. S., Pearson, G. J., Yates, G. M., Stone, J. A., and Ur, E. 2013. "2012 update of the Canadian Cardiovascular Society guidelines for the diagnosis and treatment of dyslipidemia for the prevention of cardiovascular disease in the adult." The Canadian Journal of Cardiology 29 (2): 151-67.

[5] Platt, R. 2000. "Current Concepts in Optimum Nutrition for Cardiovascular Disease." Preventive cardiology 3 (2): 83-7.

[6] Ekor, M. 2014. "The Growing Use of Herbal Medicines: Issues Relating to Adverse Reactions and Challenges in Monitoring Safety." Frontiers in Pharmacology 10 (4): 177.

[7] Yetley, E. A. 2007. "Multivitamin and Multimineral Dietary Supplements: Definitions, Characterization, Bioavailability, and Drug Interactions." The American Journal of Clinical Nutrition 85 (1): 269S-276S.

[8] Cicero, A. F., Rovati, L. C., and Setnikar, I. 2007. "Eulipidemic Effects of Berberine Administered Alone or in Combination with Other Natural Cholesterol-Lowering Agents. A Single-blind Clinical Investigation.” Arzneimittelforschung 57 (1): 26-30.

[9] Trimarco, B., and Benvenuti, C. 2009. "Controlled Clinical Studies on a New Dietary Supplement Active on Cholesterol and Triglycerides in CV Risk and Metabolic Syndrome Control." Atherosclerosis Supplements Abstract 950109.

[10] Ruscica, M., Gomaraschi, M., Mombelli, G., Macchi, C., Bosisio, R., Pazzucconi, F., Pavanello, C., Calabresi, L., Arnoldi, A., Sirtori, C. R., and Magni, P. 2014. "Nutraceutical Approach to Moderate Cardiometabolic Risk: Results of a Randomized, Double-Blind and Crossover Sudy with Armolipid Plus." Journal of Clinical Lipidology 8 (1): 61-8.

[11] Brusq, J. M., Ancellin, N., Grondin, P., Guillard, R., Martin, S., Saintillan, Y., and Issandou, M. 2006.
"Inhibition of Lipid Synthesis through Activation of AMP Kinase: An Additional Mechanism for the Hypolipidemic Effects of Berberine." Journal of Lipid Research 47 (6): 1281-8.

[12] Heber, D., Yip, I., Ashley, J. M., Elashoff, D. A., Elashoff, R. M., and Go, V. L. 1999. "Cholesterol-Lowering Effects of a Proprietary Chinese Red-yeast-rice Dietary Supplement." The American Journal of Clinical Nutrition 69 (2): 231-6.

[13] Caputi A. P., and Benvenuti, C. 2008. "Armoweb Study Group Improving the Diet Efficacy in Hypercholesterolemic Subjects with Red Yeast Rice plus Policosanol." L'Internista/Medicina Clinica.it 16: 53-60.

[14] Kong, W., Wei, J., Abidi, P., Lin, M., Inaba, S., Li, C., Wang, Y., Wang, Z., Si, S., Pan, H., Wang, S., Wu, J., Wang, Y., Li, Z., Liu, J., and Jiang, J. D. 2004. "Berberine Is a Novel Cholesterol-Lowering Drug Working through a Unique Mechanism Distinct from Statins." Nature Medicine 10 (12): 1344-51.

[15] Cicero, A.F., Derosa, G. Bove, M. Imola, F. Borghi, C. and Gaddi, A. V. 2009. "Long-Term Effectiveness and Safety of a Nutraceutical Based Approach to Reduce Cholesterolemia in Statin Intolerant Subjects with and without Metabolic Syndrome." Current Topics in Nutraceutical Research 7 (3/4): 121-6.

[16] Pisciotta, L., Bellocchio, A., and Bertolini, S. 2012. "Nutraceutical Pill Containing Berberine versus Ezetimibe on Plasma Lipid Pattern in Hypercholesterolemic Subjects and Its Additive Effect in Patients with Familial Hypercholesterolemia on Stable Cholesterol-Lowering Treatment." Lipids in Health and Disease 11:123.

[17] Izzo R., de Simone G, Giudice R, Chinali M, Trimarco V, De Luca N, Trimarco B. 2010. "Effects of Nutraceuticals on Prevalence of Metabolic Syndrome and on Calculated Framingham Risk Score in Subjects with Dyslipidemia.” Journal of Hypertension 28 (7): 1482-7.

[18] Trimarco, B., Benvenuti, C., Rozza, F., Cimmino, C. S., Giudice, R., and Crispo, S. 2011. "Clinical Evidence of Efficacy of Red Yeast Rice and Berberine in a Large Controlled Study versus Diet." Mediterranean Journal of Nutrition and Metabolism 4 (2): 133-9.

[19] Persiani, S., Sala, F., Manzotti, C., Colovic, M., Donazzolo, Y., Barbetta, B., Vitalini, C., Giacovelli, G., Benvenuti, C., Rovati, L. C. 2014. "Effect of a Combination of Berberine and Monacolin on CYP Enzymes: an Oral Cocktail Interaction Study in Healthy Volunteers". Journal of Pharmacy and Pharmacology 2 (11): 660-8. doi: 10.17265/2328-2150/2014.11.004.

[20] Food and Drug Administration. 2002. "Guidance for Industry. Food-Effect Bioavailability and Fed Bioequivalence Studies." Department of Health and 


\section{in Combination in Healthy Male Volunteers}

Human Services, Food and Drug Administration, Center for Drug Evaluation and Research (CDER). Available from:

http://www.fda.gov/downloads/RegulatoryInformation/G uidances/UCM126833.pdf

[21] European Medicines Agency. 2012. "Guideline on the Investigation of Drug Interactions." Committee for Human Medicinal Products,. Available from: http://www.ema.europa.eu/docs/en_GB/document_library /Scientific_guideline/2012/07/WC500129606.pdf

[22] Fuhr, U., Jetter, A., and Kirchheiner, J. 2007. "Appropriate Phenotyping Procedures for Drug Metabolizing Enzymes and Transporters in Humans and Their Simultaneous Use in the "Cocktail" Approach". Clinical Pharmacology and Therapeutics 81 (2): 270-83.

[23] European Medicines Agency. 2010. "Guideline on the Investigation of Bioequivalence". Committee for Human Medicinal Products. Available from: http://www.ema.europa.eu/docs/en_GB/document_library /Scientific_guideline/2010/01/WC500070039.pdf

[24] Tan, X. S., Ma, J. Y., Feng, R., Ma, C., Chen, W. J., Sun, Y. P., Fu, J., Huang, M., He, C. Y., Shou, J. W., He, W. Y., Wang, Y., and Jiang, J. D. 2013. "Tissue Distribution of Berberine and Its Metabolites after Oral Administration in Rats." PLoS One 8 (10): e77969.

[25] Gupta, P. K., Gurley, B. J., Barone, G., and Hendrickson, H. P. 2010. "Clinical Pharmacokinetics and Metabolism of Berberine and Hydrastine Following an Oral Dose of Goldenseal Supplement." Planta Medica 76-P110. Congress abstract (/ejournals/abstract/10.1055/s-0030-1251872).

[26] Chen, C. H., Uang, Y. S., Wang, S. T., Yang, J. C., Lin, C. J. 2012. "Interactions between Red Yeast Rice and CYP450 Enzymes/P-Glycoprotein and Its Implication for the Clinical Pharmacokinetics of Lovastatin". Evidence-Based Complementary and Alternative Medicine 2012: 127043.

[27] Bhattaram, V. A., Graefe, U., Kohlert, C., Veit, M., Derendorf, H. 2002. "Pharmacokinetics and Bioavailability of Herbal Medicinal Products." Phytomedicine 9 (3):1-33.

[28] Rein, M. J., Renouf, M., Cruz-Hernandez, C., Actis-Goretta, L., Thakkar, S. K., and da Silva Pinto, M. 2012. "Bioavailability of Bioactive Food Compounds: A Challenging Journey to Bioefficacy." British Journal of Clinical Pharmacology 75 (3): 588-602.

[29] Diletti, E., Hauschke, D., Steinijans, V. W. 1992. "Sample Size Determination: Extended Tables for the Multiplicative Model and Bioequivalence Ranges of 0.9 to 1.11 and 0.7 to 1.43." International Journal of Clinical Pharmacology, Therapy, and Toxicology 30 (8): 287-90.

[30] Zeng, X., and Zeng, X. 1999. "Relationship between the clinical effects of berberine on severe congestive heart failure and its concentration in plasma studied by HPLC." Biomedical Chromatography 13 (7): 442-4.

[31] Brantley, S. J., Argikar, A. A., Lin, Y. S., Nagar, S., and Paine, M. F. 2014. "Herb-Drug Interactions: Challenges and Opportunities for Improved Predictions." Drug Metabolism and Disposition 42 (3): 301-17. 